\title{
Compressed sensing for radio interferometry: spread spectrum imaging techniques
}

\author{
Y. Wiaux ${ }^{a, b}$, G. Puy ${ }^{a}$, Y. Boursier ${ }^{a}$ and P. Vandergheynst ${ }^{a}$ \\ ${ }^{a}$ Institute of Electrical Engineering, Ecole Polytechnique Fédérale de Lausanne (EPFL), \\ CH-1015 Lausanne, Switzerland; \\ ${ }^{b}$ Centre for Particle Physics and Phenomenology, Université catholique de Louvain (UCL), \\ B-1348 Louvain-la-Neuve, Belgium
}

\begin{abstract}
We consider the problem of reconstruction of astrophysical signals probed by radio interferometers with baselines bearing a non-negligible component in the pointing direction. The visibilities measured essentially identify with a noisy and incomplete Fourier coverage of the product of the planar signals with a linear chirp modulation. We analyze the related spread spectrum phenomenon and suggest its universality relative to the sparsity dictionary, in terms of the achievable quality of reconstruction through the Basis Pursuit problem. The present manuscript represents a summary of recent work. ${ }^{11}$
\end{abstract}

Keywords: techniques: image processing, techniques: interferometric

\section{COMPRESSED SENSING AND INCOHERENCE}

It is well-known that a large variety of natural signals are sparse or compressible in multi-scale dictionaries, such as wavelet frames. A band-limited signal may be expressed as the $N$-dimensional vector of its values sampled at the Nyquist-Shannon rate. By definition, a signal is sparse in some basis if its expansion contains only a small number $K \ll N$ of non-zero coefficients. More generally it is compressible if its expansion only contains a small number of significant coefficients, i.e. if a large number of its coefficients bear a negligible value. The theory of compressed sensing demonstrates that, for sparse or compressible signals, a small number $M \ll N$ of random measurements, in a sensing basis incoherent with the sparsity basis, will suffice for an accurate and stable reconstruction of the signals. ${ }^{1-4,6,7}$ The compressed sensing framework proposes a global minimization problem for the signal reconstruction, called Basis Pursuit (BP). This problem regularizes the originally ill-posed inverse problem, arising from $M$ measurements for $N$ variables, by an explicit sparsity or compressibility prior on the signal. In the presence of noise, the so-called Basis Pursuit denoise $\left(\mathrm{BP}_{\epsilon}\right)$ problem is the minimization of the $\ell_{1}$ norm of the coefficients of the signal in the sparsity basis $\Psi$ under a constraint on the $\ell_{2}$ norm of the residual noise. The $\mathrm{BP}_{\epsilon}$ problem is solved by application of non-linear and iterative convex optimization algorithms. ${ }^{5,10}$

For example, random Fourier measurements of a signal sparse in real space are a particular example of a good sensing procedure in this context. The mutual coherence $\mu$ between the orthonormal Fourier basis $\mathrm{F}$ and an orthonormal sparsity basis $\Psi$ may be defined as the maximum complex modulus of the scalar product between basis vectors of the two bases. If the unit-normed basis vectors corresponding to the lines of $\mathrm{F}$ and the columns of $\boldsymbol{\psi}$ are respectively denoted by $\left\{\boldsymbol{f}_{i}\right\}_{1 \leq i \leq N}$ and $\left\{\boldsymbol{\psi}_{j}\right\}_{1 \leq j \leq N}$, the mutual coherence between the bases reads as:

$$
\mu(\mathrm{F}, \Psi) \equiv \max _{1 \leq i, j \leq N}\left|\boldsymbol{f}_{i} \cdot \boldsymbol{\psi}_{j}\right|
$$

In other words this mutual coherence identifies with the maximum complex modulus of the Fourier coefficient values of the sparsity basis vectors. It plays an essential role in the signal reconstruction quality as, for fixed $M$, the sparsity recovered increases with the mutual incoherence as $K \propto \mu^{-2}(\mathrm{~F}, \Psi)$. The incoherence is notably maximum between the Fourier basis and the real space basis identified by a sparsity matrix $\psi \equiv \Delta$ made up of unit spikes: $\mu(\mathrm{F}, \Delta)=N^{-1 / 2}$.

Y.W.: E-mail: yves.wiaux@epfl.ch 


\section{INTERFEROMETRY AND SPREAD SPECTRUM}

Aperture synthesis in radio interferometry is a powerful technique in radio astronomy, allowing observations of the sky with otherwise inaccessible angular resolutions and sensitivities. This technique dates back to more than sixty years ago. ${ }^{9}$ In this context, the portion of the celestial sphere around the pointing direction tracked by a radio telescope array during observation defines the original real signal or image probed $x$. The field of view observed is limited by an illumination function $A^{\left(t_{0}\right)}$ with a support of size identified by $t_{0} \in \mathbb{R}_{+}$. Standard interferometers are characterized by a small field of view, so that the signal and the illumination function are assumed to be planar. Considering non-polarized radiation, they respectively read as scalar functions $x(\boldsymbol{l})$ and $A^{\left(t_{0}\right)}(\boldsymbol{l})$ of the position $\boldsymbol{l} \in \mathbb{R}^{2}$, with components $(l, m)$. Each telescope pair at one instant of observation identifies a baseline defined as the relative position between the two telescopes. To each baseline $\boldsymbol{b}_{\lambda} \in \mathbb{R}^{3}$, with components $(u, v, w)$ in units of the signal emission wavelength $\lambda$, is associated one measurement called visibility. In the simplest setting one also considers baselines with negligible component $w$ in the pointing direction of the instrument. Under this additional assumption, if the signal is made up of incoherent sources, each visibility corresponds to the value of the Fourier transform of the signal multiplied by the illumination function at a spatial frequency $\boldsymbol{u} \in \mathbb{R}^{2}$, identified by the components $(u, v)$ of the baseline projection on the plane of the signal. Radio-interferometric data are thus identified by incomplete and noisy measurements in the Fourier plane. In the perspective of the reconstruction of the original image, these data define an ill-posed inverse problem.

In the present work, we raise the important problem of the dependence of the image reconstruction quality as a function of the sparsity basis, or more generally the sparsity dictionary. The larger the typical size of the waveforms constituting the dictionary in which the signal is sparse or compressible in real space, the smaller their extension in the Fourier plane, and the smaller the incoherence between the sparsity and sensing dictionaries. In the context of compressed sensing, this leads to a degradation of the reconstruction quality for a given number $M$ of random measurements.

The detailed structure of radio-interferometric measurements might actually provide a natural response to this issue. The approximation of baselines with negligible component $w$ is a key assumption in order to identify visibilities with Fourier measurements of the original signal. This approximation actually sets a strong constraint on the field of view probed by the interferometer, requiring that it is small enough, not only for the planar approximation of the signal but also to neglect the complete effect of the component $w$ in the visibilities. We relax this approximation and consider radio interferometers with small field of view and baselines with nonnegligible component $w$. Relying on the flexibility of realistic baseline distributions, we make the simplifying assumption that all baselines have the same component $w$. In this context, all visibilities indeed identify with the values of the two-dimensional Fourier transform of a complex signal obtained as the product of the original planar signal multiplied by the illumination function $A^{\left(t_{0}\right)} x(\boldsymbol{l})$ with a linear chirp modulation $C^{(w)}(|\boldsymbol{l}|)=\mathrm{e}^{\mathrm{i} \pi w|\boldsymbol{l}|^{2}}$, where the norm $|\boldsymbol{l}|$ identifies the distance to the center of the image:

$$
y(\boldsymbol{u})=C^{\left(\widehat{w)} A^{\left(t_{0}\right)}\right.} x(\boldsymbol{u}) .
$$

Note that the multiplication by the chirp modulation amounts to the convolution of the Fourier transform of the chirp with that of the original signal multiplied by the illumination function: $C^{(w) A^{\left(t_{0}\right)}} x=\widehat{C^{(w)}} \star \widehat{A^{\left(t_{0}\right)} x}$. This convolution inevitably spreads the two-dimensional sample power spectrum of the signal multiplied by the illumination function, i.e. the square modulus of its Fourier transform, while preserving its overall norm. This spread spectrum phenomenon increases the incoherence between the sparsity and sensing dictionaries.

\section{SENSING AND SPARSITY DICTIONARIES}

The band-limited functions considered are completely identified by their Nyquist-Shannon sampling on a discrete uniform grid of $N=N^{1 / 2} \times N^{1 / 2}$ points $\boldsymbol{l}_{i} \in \mathbb{R}^{2}$ in real space with $1 \leq i \leq N$. The sampled signal is denoted by a vector $\boldsymbol{x} \in \mathbb{R}^{N} \equiv\left\{x_{i} \equiv x\left(\boldsymbol{l}_{i}\right)\right\}_{1 \leq i \leq N}$ and the illumination function is denoted by the vector $\boldsymbol{A}^{\left(t_{0}\right)} \in \mathbb{R}^{N} \equiv$ $\left\{A_{i}^{\left(t_{0}\right)} \equiv A^{\left(t_{0}\right)}\left(\boldsymbol{l}_{i}\right)\right\}_{1 \leq i \leq N}$. The chirp is complex and reads as the vector $\boldsymbol{C}^{(w)} \in \mathbb{C}^{N} \equiv\left\{C_{i}^{(w)} \equiv C^{(w)}\left(\left|\boldsymbol{l}_{i}\right|\right)\right\}_{1 \leq i \leq N}$. Because of the assumed finite field of view, the functions may equivalently be described by their complex Fourier coefficients on a discrete uniform grid of $N=N^{1 / 2} \times N^{1 / 2}$ spatial frequencies $\boldsymbol{u}_{i}$ with $1 \leq i \leq N$. This grid 
is limited at some maximum frequency defining the band limit. Due to the fact that the chirp is complex, the Fourier transform of the product $C^{(w)} A^{\left(t_{0}\right)} x$ does not bear any specific symmetry property in the Fourier plane.

We assume that the spatial frequencies $\boldsymbol{u}$ probed by all telescope pairs during the observation belong to the discrete grid of points $\boldsymbol{u}_{i}$. The Fourier coverage provided by the $M / 2$ spatial frequencies probed $\boldsymbol{u}_{b}$, with $1 \leq b \leq M / 2$, can simply be identified by a binary mask in the Fourier plane equal to 1 for each spatial frequency probed and 0 otherwise. The visibilities measured may be denoted by a vector of $M / 2$ complex Fourier coefficients $\boldsymbol{y} \in \mathbb{C}^{M / 2} \equiv\left\{y_{b} \equiv y\left(\boldsymbol{u}_{b}\right)\right\}_{1 \leq b \leq M / 2}$, possibly affected by complex noise values $\boldsymbol{n} \in \mathbb{C}^{M / 2} \equiv\left\{n_{b} \equiv n\left(\boldsymbol{u}_{b}\right)\right\}_{1 \leq b \leq M / 2}$ of astrophysical or instrumental origin. Formally, the measured visibilities may equivalently be denoted by a vector of $M$ real measures $\boldsymbol{y} \in \mathbb{R}^{M} \equiv\left\{y_{r}\right\}_{1<r<M}$ consisting of the real and imaginary parts of the complex measures, affected by the corresponding real noise values $\boldsymbol{n} \in \mathbb{R}^{M} \equiv\left\{n_{r}\right\}_{1<r<M}$. Relying on the flexibility of realistic baseline distributions, we also assume that the spatial frequencies probed arise from a uniform random selection of Fourier frequencies. As for the assumption of constant $w$, this allows us to discard considerations related to specific interferometers. It also allows us to place our discussion in a setting which complies directly with the requirement of the theory of compressed sensing for random measurements.

In this discrete setting, the Fourier coverage is in general incomplete in the sense that the number of real constraints $M$ is smaller than the number of unknowns $N: M<N$. An ill-posed inverse problem is thus defined for the reconstruction of the signal $\boldsymbol{x}$ from the measured visibilities $\boldsymbol{y}$ :

$$
\boldsymbol{y} \equiv \Phi^{\left(w, t_{0}\right)} \boldsymbol{x}+\boldsymbol{n} \text { with } \Phi^{\left(w, t_{0}\right)} \equiv \operatorname{MFC}^{(w)} \mathrm{A}^{\left(t_{0}\right)},
$$

where the matrix $\Phi^{(w)} \in \mathbb{C}^{(M / 2) \times N}$ identifies the complete linear relation between the signal and the visibilities. The matrix $\mathrm{A} \in \mathbb{R}^{N \times N} \equiv\left\{A_{i j} \equiv A_{i} \delta_{i j}\right\}_{1 \leq i, j \leq N}$ is the diagonal matrix implementing the illumination function. The matrix $\mathrm{C}^{(w)} \in \mathbb{C}^{N \times N} \equiv\left\{C_{i j}^{(w)} \equiv C_{i}^{(w)} \delta_{i j}\right\}_{1 \leq i, j \leq N}$ is the diagonal matrix implementing the chirp modulation. The unitary matrix $\mathrm{F} \in \mathbb{C}^{N \times N} \equiv\left\{F_{i j} \equiv \mathrm{e}^{-2 \mathrm{i} \pi \boldsymbol{u}_{i} \cdot \boldsymbol{x}_{j}} / N^{1 / 2}\right\}_{1 \leq i, j \leq N}$ implements the discrete Fourier transform providing the Fourier coefficients. The matrix $\mathrm{M} \in \mathbb{R}^{(M / 2) \times N^{N}} \equiv\left\{M_{b j}\right\}_{1 \leq b \leq M / 2 ; 1 \leq j \leq N}$ is the rectangular binary matrix implementing the mask characterizing the interferometer. It contains only one non-zero value on each line, at the index of the Fourier coefficient corresponding to each of the spatial frequencies probed $\boldsymbol{u}$.

For the sake of simplicity and without loss of generality, we consider simple astrophysical signals sparse in a dictionary of Gaussian waveforms, all with equal and fixed size given by a standard deviation $t \in \mathbb{R}_{+}$. The sparsity dictionary identifying Gaussian waveforms of size $t$ may be denoted by $\Psi \equiv \Gamma^{(t)}$. The sensing dictionary as seen from the sparsity dictionary itself therefore reads as $\Theta^{\left(w, t_{0}, t\right)} \equiv \Phi^{\left(w, t_{0}\right)} \Gamma^{(t)} \equiv \operatorname{MFC}^{(w)} A^{\left(t_{0}\right)} \Gamma^{(t)}$.

Let us emphasize the fact that the sparsity dictionary is obviously not an orthogonal basis. Moreover, the sensing dictionary does not correspond anymore exactly to a random selection of vectors in an orthogonal basis. No precise compressed sensing result relating sparsity and mutual coherence between such dictionaries was yet obtained. However, in the line of first results already proved with redundant dictionaries, ${ }^{8}$ one can intuitively conjecture the same trade-off between sparsity and mutual coherence as for orthogonal bases. In the perspective of assessing the $\mathrm{BP}_{\epsilon}$ reconstruction quality, it is therefore essential to understand how the mutual coherence between the sensing and sparsity dictionaries depends on the size $t$ of the Gaussian waveforms and on the chirp rate $w$.

\section{MUTUAL COHERENCE}

We assume a Gaussian illumination function $A^{\left(t_{0}\right)}$ with a size identified by a standard deviation $t_{0}$. After normalization of the vectors of the sparsity and sensing dictionaries in $\ell_{2}$ norm, a simple analytical computation gives the mutual coherence between the sensing and sparsity dictionaries as:

$$
\mu\left(\mathrm{FC}^{(w)} \mathrm{A}^{\left(t_{0}\right)}, \Gamma^{(t)}\right)=\frac{2 t t_{0}}{t^{2}+t_{0}^{2}}\left[1+\left(\frac{2 \pi w t^{2} t_{0}^{2}}{t^{2}+t_{0}^{2}}\right)^{2}\right]^{-\frac{1}{2}} .
$$

Note that one can formally re-organise the decomposition of the matrix $\Theta^{\left(w, t_{0}, t\right)}$ into modified sensing and sparsity dictionaries, respectively $\tilde{\Phi} \equiv \mathrm{MF}$ and $\tilde{\Psi}^{\left(w, t_{0}, t\right)} \equiv \mathrm{C}^{(w)} \mathrm{A}^{\left(t_{0}\right)} \Gamma^{(t)}$. In this perspective, the mutual coherence 
$\mu\left(F C^{(w)} \mathrm{A}^{\left(t_{0}\right)}, \Gamma^{(t)}\right)$ identifies with the maximum complex modulus of the Fourier coefficient values of the modified sparsity dictionary vectors, which depend on the chirp modulation and on the illumination function. Consequently, the requirement for a lower coherence can be fulfilled by an operation that spreads the two-dimensional sample power spectrum of the signal in the Fourier plane, while preserving its norm. The relation (4) is valid both for finite $N$ as well as in the continuous limit $N \rightarrow \infty$. In this continuous limit, analyzing the evolution of the coherence as a function of the parameters $t, t_{0}$, and $w$ is very enlightening.

Firstly, we consider finite non-zero values of the size $t_{0}$ of the illumination function and of the chirp rate $w$. When the size $t$ of the Gaussian waveforms tends to zero, the mutual coherence tends to zero:

$$
\lim _{t \rightarrow 0} \mu\left(\mathrm{FC}^{(w)} \mathrm{A}^{\left(t_{0}\right)}, \Gamma^{(t)}\right)=0 \text { for all } w, t_{0} \in \mathbb{R}_{+} .
$$

In the absence of chirp modulation and illumination function, this null value is expected as the modified sparsity dictionary identifies with the Dirac basis. But we also show that the mutual coherence gets to this minimum value independently of the chirp rate $w$ in this limit, and less importantly independently of the size of the illumination function $t_{0}$.

Secondly, we consider finite non-zero values of the size $t_{0}$ of the illumination function and of the size $t$ of the Gaussian waveforms, with $t<t_{0}$. In the absence of chirp, i.e. for $w=0$, the coherence is strictly positive. The finite size $t$ of a Gaussian waveform implies a Gaussian profile of its Fourier transform with a standard deviation $(2 \pi t)^{-1}$. The larger $t$, the smaller the extent of the two-dimensional sample power spectrum in the Fourier plane, and the larger the coherence. As discussed, in the presence of a chirp the spectrum is spread by means of the corresponding convolution in the Fourier plane, while the norm of the signal multiplied by the illumination function is preserved. This spread spectrum phenomenon reduces the coherence, which should enhance the quality of the $\mathrm{BP}_{\epsilon}$ reconstruction in the context of compressed sensing. In the limit of an infinite chirp rate, the coherence also tends to its minimum null value independently of the size $t$ of the Gaussian waveforms:

$$
\lim _{w \rightarrow \infty} \mu\left(\mathrm{FC}^{(w)} \mathrm{A}^{\left(t_{0}\right)}, \Gamma^{(t)}\right)=0 \text { for all } t, t_{0} \in \mathbb{R}_{+} .
$$

This limit provides a strong result in the sense that the incoherence lost by considering a sparsity dictionary of Gaussian waveforms of arbitrary non-zero size $t$ may be completely recovered thanks to a chirp modulation with high enough chirp rate $w$. This result suggests the universality of the spread spectrum phenomenon according to which the quality of the $\mathrm{BP}_{\epsilon}$ reconstruction can be rendered independent of the sparsity dictionary for a large enough component $w$ of the baselines.

\section{SIMULATIONS AND RESULTS}

The signals $x$ considered are built as the superposition of $K=10$ Gaussian waveforms, with random positions, and random central value in the interval $[0,1]$ in some arbitrary intensity units (iu). The Gaussian illumination function $A^{\left(t_{0}\right)}$ is taken with a full width at half maximum (FWHM) of $30 \mathrm{arcmin}$, corresponding to a standard deviation $t_{0} \simeq 13$ arcmin. The illumination function is centered on the signal, with unit value at the center. The signals are sampled as images $\boldsymbol{x}$ with $N=64 \times 64=4096$ pixels on a field of view of $L=L^{1 / 2} \times L^{1 / 2}=$ 30 arcmin $\times 30$ arcmin. For such fields of view the planar approximation for the signal is valid. Baselines with constant component in the pointing direction are considered, with values of the component $w$ in agreement with the constraint ensuring the validity of relation (2) for the visibilities. Observations are considered for $M / 2$ complex visibilities with $M / 2 \in\{50,100,200,300,400,500,1000\}$. A small amount of instrumental noise is also added as independent identically distributed Gaussian noise.

The size $t$ of the Gaussian waveforms may be written in terms of a discrete size $t_{d}$ as $t=t_{d} L^{1 / 2} / \pi N^{1 / 2}$. We consider the values $t_{d}=2$ and $t_{d}=16$. For $t_{d}=2$ the approximate band limit of the signal is around the same value as that accessible on the grids considered, while for $t_{d}=16$ it is much smaller. Original signal samples are reported in Fig. 1. The component $w$ may also be written in terms of a discrete component $w_{d}$ as $w=w_{d} N^{1 / 2} / L$. We consider the values $w_{d}=0$ and $w_{d}=1$. The case $w_{d}=0$ identifies baselines with negligible component $w$. The case $w_{d}=1$ identifies baselines with non-negligible and constant component $w$, corresponding to a linear 

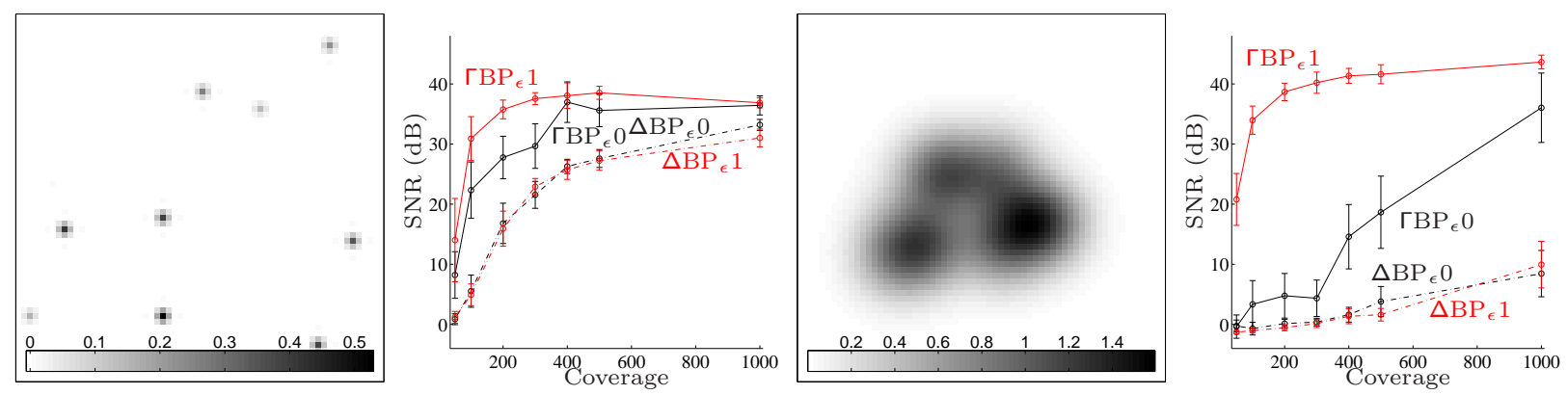

Figure 1. The extreme left and center right panels represent original signal samples for $t_{d}=2$ and $t_{d}=16$ respectively. The center left and extreme right panels represent the SNR of the reconstructions as a function of the coverage identified by the number of complex visibilities $M / 2 \in\{50,100,200,300,400,500,1000\}$ for $t_{d}=2$ and $t_{d}=16$ respectively.

chirp modulation with a band limit equal to that accessible on the grids. In other words the component $w$ is as large as the maximum value of the components $u$ and $v$ in the plane of the signal.

A number of 30 simulations is generated for each value of $t_{d}$ and $w_{d}$ considered. The visibilities are simulated, and the signals are reconstructed through the $\mathrm{BP}_{\epsilon}$ problem, which is solved by convex optimization. The quality of the reconstructions is analyzed in terms of the signal-to-noise ratio SNR $\equiv-20 \log _{10}\left(\Sigma^{\left(\mathrm{A}^{\left(t_{0}\right)}(\boldsymbol{x}-\overline{\boldsymbol{x}})\right)} / \Sigma^{\left(\mathrm{A}^{\left(t_{0}\right)} \boldsymbol{x}\right)}\right)$, where $\Sigma^{\left(\mathrm{A}^{\left(t_{0}\right)} x\right)}$ stands for the sample standard deviation of the original signal multiplied by the illumination function $\mathrm{A}^{\left(t_{0}\right)} \boldsymbol{x}$, and $\Sigma^{\left(\mathrm{A}^{\left(t_{0}\right)}(\boldsymbol{x}-\overline{\boldsymbol{x}})\right)}$ for that of the discrepancy signal $\mathrm{A}^{\left(t_{0}\right)}(\boldsymbol{x}-\overline{\boldsymbol{x}})$.

We actually compare two different settings for the reconstructions. In the first setting, called $\Gamma \mathrm{BP}_{\epsilon}$, we assume that the Gaussian waveform dictionary with appropriate $t$ is known, and we use it explicitly as sparsity dictionary: $\Psi \equiv \Gamma^{(t)}$. As a consequence, the $\Gamma \mathrm{BP}_{\epsilon}$ problem deals with the best possible sparsity value $K=10$. The reconstructions in the absence $\left(w_{d}=0\right)$ and in the presence $\left(w_{d}=1\right)$ of the chirp modulation are respectively denoted by $\Gamma \mathrm{BP}_{\epsilon} 0$ and $\Gamma \mathrm{BP}_{\epsilon} 1$. It is the precise setting in which we just brought up the spread spectrum phenomenon and its universality on the basis of considerations relative to the mutual coherence between the sensing and sparsity dictionaries. In the second setting, called $\Delta \mathrm{BP}_{\epsilon}$, we assume that the sparsity dictionary is the real space basis: $\Psi \equiv \Delta$. As a consequence, the $\Delta \mathrm{BP}_{\epsilon}$ problem deals with the best possible coherence value. However, the sparsity computed in real space increases drastically with the size of the Gaussian waveforms, suggesting that the reconstruction quality should clearly decrease when the Gaussian size increases. The reconstructions in the absence $\left(w_{d}=0\right)$ and in the presence $\left(w_{d}=1\right)$ of the chirp modulation are respectively denoted by $\triangle \mathrm{BP}_{\epsilon} 0$ and $\Delta \mathrm{BP}_{\epsilon} 1$. But the mutual coherence here remains unaffected by the chirp modulation, so that this modulation should fail to enhance the reconstruction quality in this case.

The results of the analysis are reported in Fig. 1. The curves represent the mean SNR of the reconstructions over the 30 simulations, and the vertical lines identify the error at 1 standard deviation.

Firstly, for each size $t_{d}$ of the Gaussian waveforms and each reconstruction procedure, the SNR obviously benefits from an increase in the number $M / 2$ of visibilities measured, corresponding to an increase in the information directly available on the signal.

Secondly, in the absence of chirp it clearly appears that, for each size $t_{d}$ of the Gaussian waveforms and each number of visibilities measured $M / 2$, the $\Gamma \mathrm{BP}_{\epsilon} 0$ reconstruction exhibits a significantly better SNR than the $\triangle \mathrm{BP}_{\epsilon} \mathrm{O}$ reconstruction. This suggests that for the signals considered it is better to optimize the sparsity by accounting for the proper dictionary, than to optimize the mutual coherence by formally postulating that the signal lives in real space. It also appears that, for each number of visibilities measured $M / 2$, the SNR of both kinds of reconstructions significantly degrades when the size $t_{d}$ of the Gaussian waveforms increases. This behaviour is due to the fact that either the mutual coherence, in the case of $\left\lceil\mathrm{BP}_{\epsilon} 0\right.$, or the sparsity, in the case of $\triangle \mathrm{BP}_{\epsilon} 0$, get farther from their optimal values.

Thirdly, the SNR of the $\triangle \mathrm{BP}_{\epsilon} 0$ and $\triangle \mathrm{BP}_{\epsilon} 1$ reconstructions remain undistinguishable from one another at 1 standard deviation, independently of the size $t_{d}$ of the Gaussian waveforms and of the number of visibilities 
measured $M / 2$. This illustrates the fact that the mutual coherence is already optimal in the absence of chirp modulation, so that any chirp modulation will fail to enhance the reconstruction quality.

Finally, for any number of visibilities measured $M / 2$ and for the size $t_{d}=16$ of the Gaussian waveforms, the SNR of the $\Gamma \mathrm{BP}_{\epsilon} 1$ is significantly larger than that of the $\left\lceil\mathrm{BP}_{\epsilon} 0\right.$ reconstruction. This is the spread spectrum phenomenon related to the reduction of the mutual coherence in the presence of the chirp modulation. Moreover, the SNR of the $\Gamma \mathrm{BP}_{\epsilon} 1$ reconstruction is essentially independent of the sparsity dictionary identified by $t_{d}$. This supports very strongly the principle of universality of the spread spectrum phenomenon relative to the sparsity dictionary, in perfect agreement with our theoretical considerations. The reconstruction quality also appears to be more stable around the mean SNR values in the presence of the chirp modulation.

\section{CONCLUSION}

The reconstruction quality of signals from radio-interferometric measurements might be rendered independent of the sparsity dictionary, provided that the interferometers may be designed to ensure large enough components of the baselines in the pointing direction.

\section{ACKNOWLEDGMENTS}

Y. W. is Postdoctoral Researcher of the Belgian National Science Foundation (F.R.S.-FNRS). Y. B. a Postdoctoral Researcher funded by the APIDIS European Project.

\section{REFERENCES}

[1] Baraniuk R., 2007, IEEE Signal Process. Mag., 24, 118

[2] Candès E. J., 2006, in Sanz-Solé M., Soria J., Varona J. L., Verdera J., eds, Proc. Int. Congress Math. Vol. 3. Euro. Math. Soc. Zürich, p. 1433

[3] Candès E. J., Romberg J., Tao T., 2006a, IEEE Trans. Inf. Theory, 52, 489

[4] Candès E. J., Romberg J., Tao T., 2006b, Comm. Pure and Appl. Math., 59, 1207

[5] Combettes P. L., Pesquet J.-C., 2007, IEEE Sel. Top. Signal Process., 1, 564

[6] Donoho D. L., 2006, IEEE Trans. Inf. Theory, 52, 1289

[7] Donoho D. L., Tanner J., 2009, J. Amer. Math. Soc., 22, 1

[8] Rauhut H., Schnass K., Vandergheynst P., 2008, IEEE Trans. Inf. Theory, 54, 2210

[9] Thompson A. R., Moran J. M., Swenson G. W. Jr, 2004, Interferometry and Synthesis in Radio Astronomy. WILEY-VCH Verlag GmbH \& Co. KGaA, Weinheim

[10] van den Berg E., Friedlander M. P., 2008, SIAM J. Sci. Comput., 31, 890

[11] Wiaux Y., Puy G., Y. Boursier, Vandergheynst P., 2009, preprint (arXiv:0907.0944v1 [astro-ph.IM]) 